\title{
Research
}

\section{Does a decision aid help physicians to detect chronic obstructive pulmonary disease?}

\begin{abstract}
\section{Background}

Numerous decision aids have been developed recently, but the value they add above that of the initial clinical assessment is not well known
\end{abstract}

\section{Aim}

To quantify whether a formal decision aid for chronic obstructive pulmonary disease (COPD) adds diagnostic information, above the physician's clinical assessment.

\section{Design and setting}

Subanalysis of a diagnostic study in Dutch primary care.

\section{Method}

Sixty-five primary care physicians included 357 patients who attended for persistent cough and were not known to have COPD. The physicians estimated the probability of COPD after short history taking and physical examination. After this, the presence or absence of COPD was determined using results of extensive diagnostic work-up. The extent to which an 8 item decision aid for COPD, which included only symptoms and signs, added diagnostic value above the physician's estimation was quantified by the increase of the area under the receiver operating characteristic curve (ROC area), and the improvement in diagnostic risk classification across two classes: "low probability of COPD' $(<20 \%)$ and 'possible COPD' $(\geq 20 \%)$.

\section{Results}

One hundred and four patients (29\%) had COPD. Adding the decision aid to the clinical assessment increased the ROC area from 0.75 (95\% confidence interval $[\mathrm{Cl}]=0.70$ to 0.81$)$ to $0.84(95 \% \mathrm{Cl}=0.80$ to 0.89$)(P<0.005)$, and improved the diagnostic risk classification of the patients, such that 35 fewer patients needed spirometry testing and eight fewer COPD cases were missed.

\section{Conclusion}

A short decision aid for COPD added diagnostic value to the physician's clinical assessment.

\section{Keywords}

COPD; cough; diagnosis; diagnostic accuracy; primary care.

\section{INTRODUCTION}

The diagnostic process for chronic obstructive pulmonary disease (COPD) in primary care starts with history taking and physical examination, and if results remain suggestive of COPD, spirometry is performed to diagnose or exclude COPD. Diagnosing or excluding COPD, especially the early cases, is not straightforward, as there is no single 'gold standard' available., 1,2 Spirometry is very important, but results can only be interpreted in combination with symptoms and signs. There is substantial underdiagnosis of COPD in primary care,,4 and awareness of possible COPD in symptomatic patients is recommended. However to perform spirometry in all patients with respiratory complaints is neither efficient nor feasible. ${ }^{2}$ Recently, the authors developed a decision aid for COPD, using only symptoms and signs, to assist physicians in estimating the probability of COPD and enhance efficient use of spirometry. The aid, developed from the data of 400 patients consulting their primary care physician with persistent cough, showed good ability to exclude COPD (negative predictive value $>90 \%$ ) (BDL Broekhuizen et al, unpublished data, 2010).

However, when a patient presents with a general symptom like persistent cough, the diagnostic process in practice usually does not start with obtaining the result of a formal decision aid. Rather, physicians first

BDL Broekhuizen, MD, $\mathrm{PhD}$, researcher, department of general practice; APE Sachs, MD $\mathrm{PhD}$, associate professor, department of general practice; KJM Janssen, $\mathrm{PhD}$, assistant professor, department of clinical epidemiology; G-J Geersing $M D$, researcher, department of general practice; KGM Moons, PhD, professor department of clinical epidemiology; AW Hoes, MD, PhD, professor, department of general practice and clinical epidemiology; TJM Verheij, MD, PhD, professor, department of general practice, University Medical Center Utrecht, the Netherlands. explore the problem using intuitive short history taking and physical examination. This results in an initial assessment of the probability of a possible underlying disorder. After this, a decision aid is more likely to be used to obtain more diagnostic certainty. Therefore, to determine whether an 8-item decision aid for COPD is potentially useful for practice, its diagnostic accuracy should be determined in addition to the (initial) physician's assessment.

Despite the large number of decision aids published each year, 5,6 little is known about the added value of decision aids above the physician's assessment. A few studies, of disorders other than COPD, compared the physician's assessment with a decision aid. In some the aid was superior, ${ }^{7-9}$ and in others the physician's assessment was. ${ }^{10,11}$ However the added value of the decision aid was not quantified

The aim of the present analysis was to determine whether the recently developed 8-item decision aid for COPD added diagnostic value to the physician's clinical assessment, in middle-aged and older primary care patients presenting with persistent cough.

\section{METHOD}

\section{Design and study population}

This was a subanalysis of a large Dutch diagnostic study on COPD performed from 2006 to 2009. The study protocol has been

\section{Address for correspondence}

Berna DL Broekhuizen, University Medical Center Utrecht, Julius Center for Health Sciences and Primary Care, PO Box 85500, 3508 GA Utrecht, the Netherlands.

E-mail: b.d.l.broekhuizendumcutrecht.nl.

Submitted: 1 November 2010; Editor's response: 11 January 2011; final acceptance: 18 March 2011. CBritish Journal of General Practice

This is the full-length article (published online 26 Sep 2011) of an abridged version published in print. Cite this article as: $\mathbf{B r} \mathbf{J}$ Gen Pract 2011. DOI: 10.3399/bjgp11X601398. 


\section{How this fits in}

Numerous decision aids have been developed in recent decades. Decision aids for general complaints like cough are usually applied only after the clinical assessment of the physician. Whether decision aids have added diagnostic value to this clinical assessment was unknown This study found that a short decision aid for chronic obstructive pulmonary disease (COPD) that included only symptoms and signs added relevant diagnostic value to the physician's clinical assessment, in 357 patients presenting with persistent cough. These results suggest that implementation of diagnostic decision tools in the diagnostic pathway for COPD could enhance efficient use of spirometry.

described in detail elsewhere. ${ }^{4}$ Briefly, inclusion criteria for patients were being aged over 50 years and consulting their primary care physician for complaints of cough for 14 days or longer. Exclusion criteria were known COPD, suspected pneumonia, and terminal illness.

\section{Physician's assessment and a decision aid for COPD}

On the day the patient presented with persistent cough, the physician, who was the GP of the patient, recorded the results of short standardised history taking and physical examination, and, directly thereafter, their estimation of the probability that the patient had COPD.

All patients then underwent extensive further diagnostic work-up, including repeated spirometry by the GP, and secondary-care lung function testing lbody plethysmography and carbon monoxide diffusing capacity measurement). Finally, an expert panel determined the presence or absence of COPD (reference test), according to international guidelines, ${ }^{1}$ using the results of all documented study tests, including the results of history taking and physical examination, except the GPestimated probability of COPD. The expert panel also determined the severity of COPD according to the criteria of the Global Initiative for Chronic Obstructive Lung
Disease (GOLD), ${ }^{1}$ and possible other diagnoses, like asthma.

Previously, the authors developed a decision aid for COPD including the independently contributing items of history taking and physical examination (BDL Broekhuizen et al, unpublished data, 2010). This aid was developed from the same study patients as included for the present analysis, using multivariable logistic regression analysis, with the expert panel diagnosis (COPD or no COPD) as diagnostic outcome. The final 8-item decision aid was internally validated by bootstrapping techniques to adjust the regression coefficients for overfitting and is presented in Box 1.

\section{Analysis}

In the original diagnostic study, 400 patients with persistent cough were included. In the present analysis, patients were included if the physician's clinical assessment and the panel diagnosis were available, resulting in 357 participants, included (and assessed) by 65 GPs (Figure 1). The physician's estimation of the probability of COPD was entered as a continuous variable in a multivariable logistic regression analysis, with COPD as diagnostic outcome. The estimated probability for COPD by the previously developed decision aid was added as a continuous variable. To quantify the added discriminative value of the decision aid above the physician, the area under the receiver operating characteristic curve (ROC area) of the physician's estimate and of the combination of the physician's estimate and the decision aid, were compared.

Next, the extent to which the addition of the decision aid resulted in improvement in diagnostic risk classification across clinically relevant risk groups was quantified. In daily practice, the main aim of history taking and physical examination in cases of possible COPD, is to distinguish patients in whom COPD can safely be excluded (low estimated probability), from those who should undergo spirometry. Therefore, two predefined risk classes were used: 'low probability of COPD' $(<20 \%)$ and 'possible COPD' ( $\geq 20 \%)$. As there are no generally accepted thresholds for these risk

\section{Box 1. Decision aid for COPD that was developed in $\mathbf{4 0 0}$ patients with persistent cough ${ }^{3}$}

Risk of COPD presence $=1 /(1+\exp -[-5.6+0.2 \times$ every 5 years above age of $50+0.6 \times$ male sex $+1.0 \times$ current smoking $+0.7 \times>20$ pack years $+0.6 \times$ cardiovascular disease $+0.6 \times$ complaints of wheezing $+0.7 \times$ diminished breath sounds]) 


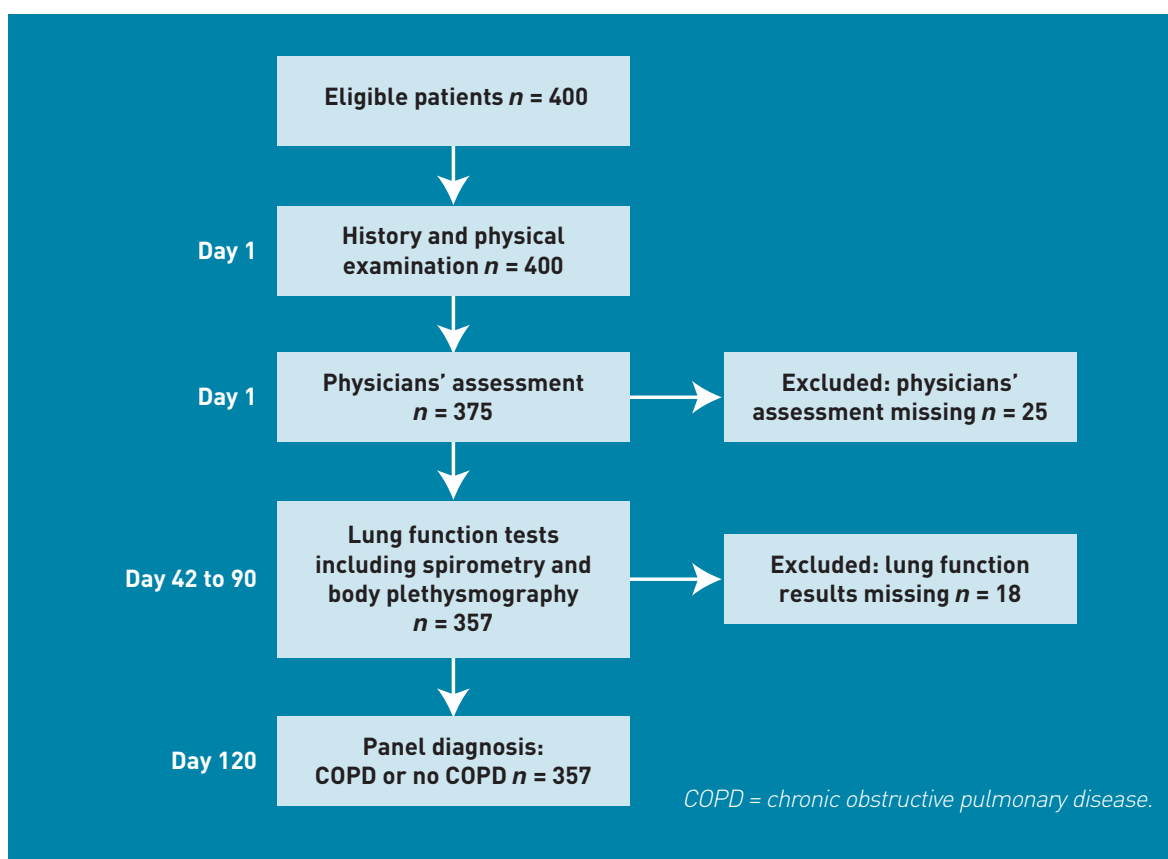

Figure 1. Flow chart of the study and participants.

\section{Table 1. Demographic and clinical characteristics of the 357 study patients}

\begin{tabular}{lc} 
Characteristic & \\
\hline Demographics (day 1) & 63 (9) \\
Mean age, years (SD) & 46 \\
Male sex, \% & 28 \\
\hline History taking (day 1) & 50 \\
Current smoker, \% & 102 (40) \\
Complaints of wheezing, \% & 14 \\
Mean duration of cough, days (median) & 12 \\
\hline Physical examination (day 1) & $0.41(0.27)$ \\
Pulmonary wheezing, \% & 100 (19) \\
Diminished breath sounds, \% & 73 (10) \\
\hline Clinical view physician (day 1) & 29 \\
Estimated probability of COPD, mean (SD) & \\
\hline Lung function tests (day 90) & \\
Post-dilator FEV $1, \%$ predicted mean (SD) & \\
Post-dilator FEV $/$ FVC, mean (SD) & Panel diagnosis \\
COPD present, \% & FEV = forced expiratory volume in 1 second. FVC = forced vital capacity. SD = standard deviation.
\end{tabular}

( $n=102)$ were current smokers (Table 1). One hundred and four participants had COPD (prevalence 29\%), of which 73 were mild lforced expiratory volume in 1 second $\left[\mathrm{FEV}_{1}\right]>80 \%$ predicted), 28 moderate (FEV $50-80 \%$ predicted), and three severe (FEV $<50 \%$ predicted). Other diagnoses were asthma in 50 participants (in 16 participants this was known before the studyl, of which 19 had both COPD and asthma, four had heart failure, and one had lung cancer. Characteristics of the analysed and excluded $(n=43)$ subjects did not substantially differ.

The ROC area of a regression model including only the physician's estimate was $0.75(95 \%$ confidence interval $[\mathrm{Cl}]=0.70$ to 0.81). Adding the decision aid significantly increased the ROC area (Figure 2) to 0.84 $(95 \% \mathrm{Cl}=0.80$ to 0.89$)(P<0.005)$

Diagnostic classification by the physician is shown in Figure 3: of the 138 subjects estimated with low probability, 119 actually had no COPD, resulting in a negative predictive value (NPV) of low estimated probability of $86 \%$. Of the 219 patients with a high $(\geq 20 \%)$ estimated probability (possible COPD), 85 really had COPD, rendering a positive predictive value (PPV) of $39 \%$ for a high estimated probability. Diagnostic classification by the combination of the physician's assessment and the decision aid resulted in a NPV of $94 \%$ and a PPV of $51 \%$ for, respectively, low and high estimated probability, and implied that 35 fewer patients underwent spirometry (184 instead of 219) and eight fewer COPD cases were missed (11 instead of 19) (Figure 3). Using a threshold of $10 \%$ and $30 \%$ for low probability (to exclude COPD), resulted in, respectively, 37 fewer patients undergoing spirometry (253 instead of 290) and three fewer missed COPD cases (two instead of five), and 27 fewer patients undergoing spirometry (147 instead of 174) and nine fewer missed cases (20 instead of 29) (results not shown).

\section{DISCUSSION}

\section{Summary}

The diagnostic value of a aid including 8 items of history taking and physical examination in addition to the physician's assessment, was studied in patients presenting with persistent cough. The aid added diagnostic value (ROC area increased from $0.75[95 \% \mathrm{Cl}=0.70$ to 0.81$]$ to 0.84 [95\% $\mathrm{Cl}=0.80$ to 0.89$]$ ) and improved the diagnostic risk classification of the patients, such that 35 fewer patients needed spirometry testing and eight fewer COPD cases were missed. 
Figure 2. Receiver operating characteristic curves of the physician's assessment and the physician's assessment plus the decision aid's estimate combined.

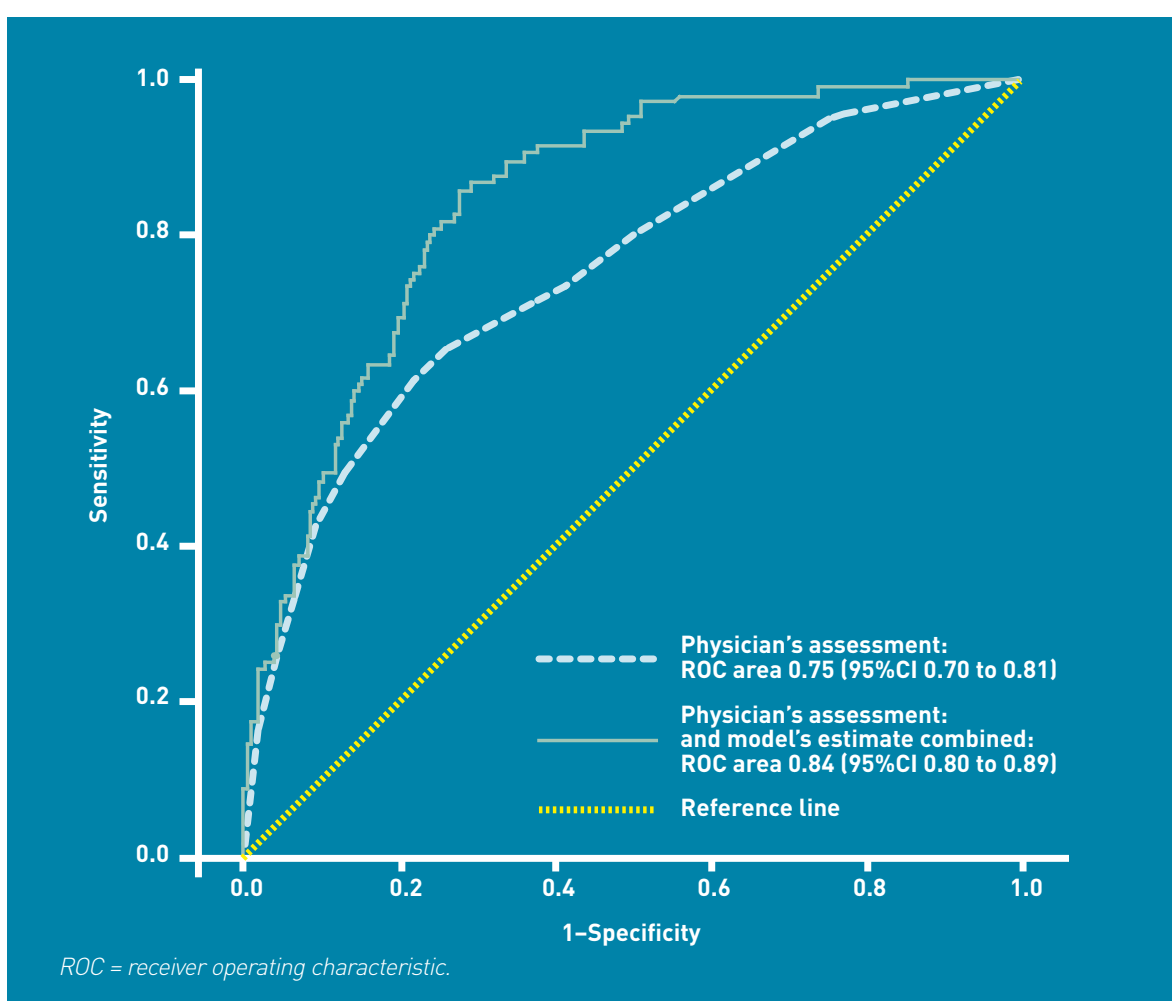

\section{Strengths and limitations}

To appreciate the present results, a few potential strengths and weaknesses of the study should be addressed. First, a strength of the study was the valid measurement of the physician's initial clinical assessment, directly after short standard history taking and physical examination, without knowledge on spirometry results or the diagnostic probability estimated by the aid. Moreover, all patients underwent the reference test for COPD, irrespective of the physician's or aid's estimation, eliminating verification bias.

A limitation is that incorporation bias was possibly induced by presenting the results of history taking and physical examination to the expert panel to decide on the presence or absence of COPD (the reference test). These items were included in the reference test to prevent misclassification of COPD. Nevertheless, the effect of incorporation bias was probably limited because the expert panel decision was based on many more items, of which, for example, the lung functions tests probably had much more influence on the outcome of the reference test. A second limitation is that the applied decision aid had been internally validated, but not externally, that is, in new patients, which would have resulted in a more generalisable decision aid. ${ }^{12}$ Finally, in the present study, physicians were requested to report their estimated probability of the presence of COPD in their patients visiting with cough, while it is possible that they might otherwise not even have considered this diagnosis. Therefore, the physician's estimation in the study may have been more accurate than the initial diagnostic assessment of COPD in daily practice.

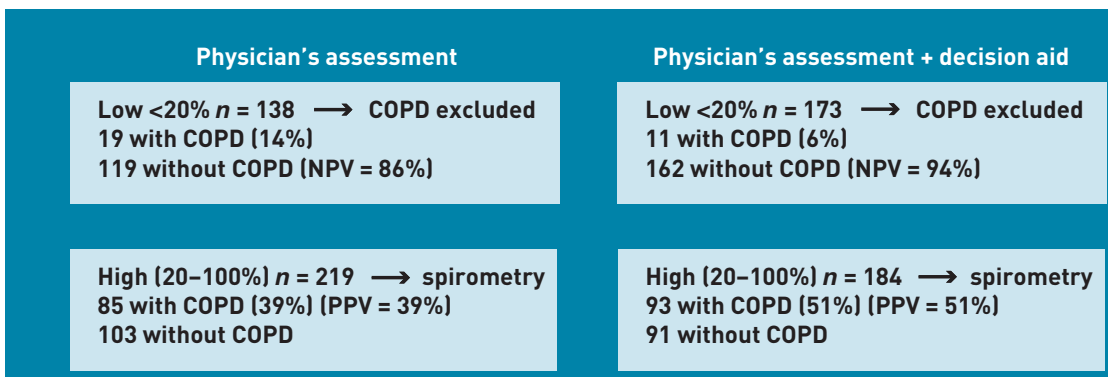




\section{Comparison with existing literature}

Others have studied the diagnostic value of clinical assessment for COPD, ${ }^{13-17}$ and reported sensitivities ranging from $50 \%$ to $64 \%$ and specificities from $64 \%$ to $93 \%$. Considering an estimated probability of $<20 \%$ as 'COPD excluded' in the present study, would result in a sensitivity of $82 \%$ and a specificity of $47 \%$. However, the other studies did not include primary care patients, and COPD severity was mostly severe; therefore results are difficult to compare with the present ones. To the authors' knowledge, no studies have quantified the diagnostic value added to the physician's clinical assessment by a decision aid for COPD.

In this study, the clinical assessment remained independently associated with COPD after addition of the decision aid's estimate. Hence, the clinical assessment incorporated information not included in the variables of the decision aid. Other studies have also reported, for other disorders than COPD, that the clinical assessment was an independent diagnostic tool. 18,19 This finding is sometimes referred to as a physician's 'gut feeling', which is obviously hard to define or to measure. ${ }^{20}$ Underlying factors of this gut feeling could be awareness of other risk factors for COPD, knowledge about prior respiratory complaints, or noticing other suggestive clinical findings during history taking and physical examination that were not registered in the present study.

\section{Implications for practice and research}

The implications of the added value of a decision aid - fewer spirometries needed and more detected cases (that is, fewer missed cases) of COPD - are relevant for daily practice. Cough is one of the most frequent complaints in primary care, which underlines the need for efficient use of spirometry. Spirometry is safe, but time consuming and costly, because it must be performed by trained professionals.

A debatable issue is the willingness of physicians to implement decision aids in daily practice. ${ }^{21,22}$ Most of the numerous decision aids or models published in recent decades, have never been implemented in daily practice. In the present study, results of the clinical assessment and the decision aid were combined, assuming that the aid was always used, and that reclassification of diagnostic risk was always applied by physicians to decide on whether or not to perform spirometry. However, physicians are usually more inclined to use a decision aid if they are not confident enough about the presence or absence of the target disorder after short history taking and physical examination. ${ }^{23}$ Moreover, according to the widely acknowledged cognitive mechanisms of 'conservatism' and 'anchoring', physicians who suspect (the absencel of a certain disorder, especially a severe disorder, are less willing to alter their diagnostic risk assessment, despite controversial new information, than those who do not have this strong suspicion..$^{23-25}$ An implementation study could provide evidence on how clinical decision making is really altered by the use of a decision aid, in patients with possible COPD. ${ }^{22}$
University Medical Center Utrecht approved the study. The ethics committee of the University Medical Centre Utrecht approved the study (protocol number 05/142).

\section{Provenance}

Freely submitted; externally peer reviewed.

\section{Competing interests}

Alfred PE Sachs has been speaker on congresses, sponsored by GlaxoSmithKline, Boehringer-Ingelheim, Pfizer, or AstraZeneca. Theo JM Verheij participates in studies that have received grants from GlaxoSmithKline and Wyeth. All other authors have declared no competing interests.

\section{Acknowledgements}

We gratefully acknowledge all participating patients, GPs, and other healthcare professionals who contributed to the study.

\section{Discuss this article}

Contribute and read comments about this article on the Discussion Forum:

http://www.rcgp.org.uk/bjgp-discuss 


\section{REFERENCES}

1. Rabe KF, Hurd S, Anzueto A, et al. Global strategy for the diagnosis, management, and prevention of chronic obstructive pulmonary disease: GOLD executive summary. Am J Respir Crit Care Med 2007; 176(6): 532-555.

2. Levy ML, Quanjer PH, Booker R, et al. Diagnostic spirometry in primary care: Proposed standards for general practice compliant with American Thoracic Society and European Respiratory Society recommendations. Prim Care Respir J 2009; 18(3): 130-147.

3. Bednarek M, Maciejewski J, Wozniak M, et al. Prevalence, severity and underdiagnosis of COPD in the primary care setting. Thorax 2008; 63(5) 402-407

4. Broekhuizen BD, Sachs AP, Hoes AW, et al. Undetected chronic obstructive pulmonary disease and asthma in people over 50 years with persistent cough. Br J Gen Pract 2010; 60(576): 489-494.

5. Royston P, Moons KG, Altman DG, Vergouwe Y. Prognosis and prognostic research: Developing a prognostic model. BMJ 2009; 338: b604.

6. Laupacis A, Sekar N, Stiell IG. Clinical prediction rules. A review and suggested modifications of methodological standards. JAMA 1997; 277(6): 488-494.

7. Palchak MJ, Holmes JF, Kuppermann N. Clinician judgment versus a decision rule for identifying children at risk of traumatic brain injury on computed tomography after blunt head trauma. Pediatr Emerg Care 2009, 25(2): 61-65

8. Cooper J, Kapur N, Kway-Jones K. A comparison between clinicians assessment and the Manchester Self-Harm Rule: a cohort study. Emerg Med J 2007; 24(10): 720-721.

9. Perez RS, Burm PE, Zuurmond WW, et al. Physicians' assessments versus measured symptoms of complex regional pain syndrome type 1: presence and severity. Clin J Pain 2005; 21(3): 272-276.

10. Glas AS, Pijnenburg BA, Lijmer JG, et al. Comparison of diagnostic decision rules and structured data collection in assessment of acute ankle injury. CMAJ 2002; 166(6): 727-733.

11. Sinuff T, Adhikari NK, Cook DJ, et al. Mortality predictions in the intensive care unit: comparing physicians with scoring systems. Crit Care Med 2006; 34(3): 878-885
12. Justice AC, Covinsky KE, Berlin JA. Assessing the generalizability of prognostic information. Ann Intern Med 1999; 130(6): 515-524.

13. Garcia-Pachon E. Paradoxical movement of the lateral rib margin (Hoover sign) for detecting obstructive airway disease. Chest 2002; 122(2): 651-655.

14. Badgett RG, Tanaka DJ, Hunt DK, et al. The clinical evaluation for diagnosing obstructive airways disease in high-risk patients. Chest 1994; 106(5): 1427-1431.

15. McAlister FA, Straus SE, Sackett DL. Why we need large, simple studies of the clinical examination: the problem and a proposed solution. CARE-COAD group. Clinical Assessment of the Reliability of the Examination-Chronic Obstructive Airways Disease Group. Lancet 1999; 354(9191): 1721-1724.

16. Holleman DR Jr, Simel DL, Goldberg JS. Diagnosis of obstructive airways disease from the clinical examination. J Gen Intern Med 1993; 8(2): 63-68.

17. Fletcher CM. The clinical diagnosis of pulmonary emphysema; an experimental study. Proc R Soc Med 1952; 45(9): 577-584.

18. Tierney WM, Fitzgerald J, McHenry R, et al. Physicians' estimates of the probability of myocardial infarction in emergency room patients with chest pain. Med Decis Making 1986; 6(1): 12-17.

19. Stolper $E$, Van RP, Van de WM, et al. Consensus on gut feelings in general practice. BMC Fam Pract 2009; 10: 66

20. Greenhalgh T. Intuition and evidence-uneasy bedfellows? Br J Gen Pract 2002; 52(478): 395-400

21. Moons KG, Altman DG, Vergouwe Y, Royston P. Prognosis and prognostic research: application and impact of prognostic models in clinical practice. BMJ 2009; 338: b606.

22. Reilly BM, Evans AT. Translating clinical research into clinical practice: impact of using prediction rules to make decisions. Ann Intern Med 2006; 144(3): 201-209.

23. Friedman CP, Gatti GG, Franz TM, et al. Do physicians know when their diagnoses are correct? Implications for decision support and error reduction. J Gen Intern Med 2005; 20(4): 334-339.

24. Tversky A, Kahneman D. Judgment under uncertainty: heuristics and biases. Science 1974; 185(4157): 1124-1131.

25. Elstein AS, Schwartz A. Clinical problem solving and diagnostic decision making: selective review of the cognitive literature. BMJ 2002; 324(7339): 729-732. 